\title{
REPRODUCTIVE OUTCOMES AFTER ABDOMINAL MYOMECTOMY
}

\author{
Yasmin Fatima, Mumtaz Amir \\ Combined Military Hospital Multan/National University of Medical Sciences (NUMS) Pakistan
}

\begin{abstract}
Objective: To determine the frequency of reproductive outcomes after abdominal myomectomy in infertile women. Study Design: Case series.

Place and Duration of Study: at Combined Military Hospital, Multan Pakistan, from Sep 2015 to Feb 2017.

Methodology: This study included 60 women who underwent myomectomy to retain their capabilities of reproduction. Inclusion criteria were infertile women with uterine fibroids. Routine investigation was done to all patients like ultrasound and hysterosalpingogram before and after the procedure. The procedure of abdominal myomectomy was done under general anesthesia. All fibroids were enucleated and large blood vessels were tied. Vicryl no.1 was used to close the uterine defect. All patients were followed up for 3, 6 and 12 months interval and data was collected for conception, spontaneous conception, and conception with assisted reproductive technique, live birth and miscarriage.

Results: In this study mean age was $27.33 \pm 4.03$ years, mean number of fibroid was $1.366 \pm 0.48$ and mean size of fibroid was $7.06 \pm 1.64 \mathrm{~cm}$. Twenty eight $(46.7 \%)$ women had conceived after myomectomy, in which $92.9 \%$ had spontaneous conception and $7.1 \%$ by assisted reproductive technique.

Conclusion: Abdominal myomectomy should be the standard treatment of infertile women with uterine fibroids if no other underline cause of infertility. Moreover, this study results also showed that younger patients might be benefited more in term of reproductive outcomes after abdominal myomectomy.
\end{abstract}

Keywords: Abdominal myomectomy, Age, Reproductive outcomes, Uterine fibroids.

This is an Open Access article distributed under the terms of the Creative Commons Attribution License (https://creativecommons.org/licenses/by-nc/4.0/), which permits unrestricted use, distribution, and reproduction in any medium, provided the original work is properly cited.

\section{INTRODUCTION}

Uterine fibroids are benign in nature and are composed of smooth muscle of the uterus, which consist of extracellular matrix and unordered collagen. Usually they formed as multicellular cell and in multiple numbers on uterine wall in whirlpool shaped. Then they grow in size and numbers in many years, due to estrogen stimulation along with progesterone. The reason of fibroids development is unknown but it is considered that it is due to genetic changes, growth factor and hormones, at the time of menstrual cycle ${ }^{1}$. Major signs of fibroids are increased bleeding during menses along with feeling pressure on abdomen which causes impact on daily life style ${ }^{2}$.

Surgical procedures are gold standard for fibroids treatment and are costly but in return they improve the quality of life of the patient ${ }^{3}$. As hysterectomy is definitive treatment of fibroids but myomectomy is preferred by those women who want to retain uterus for pregnancy. About $50 \%$ of the infertile women conceived after myomectomy. About $75 \%$ of women got pregnant within first year of myomectomy ${ }^{4}$. The reason behind infertility due to fibroids is that leiomyomas cause damage to uterine cavity. Due to this damage, by large

Correspondence: Dr Yasmin Fatima, Classified Gynaecologist, Combined Military Hospital Zhob, Pakistan

Received: 10 Feb 2020; revised received: 17 Feb 2020; accepted: 17 Feb 2020 masses, the normal physiology of implantation along with displacement of cervix got disturb ${ }^{5}$. Due to location of fibroids in the uterine cavity it is also associated with recurrent abortions ${ }^{6}$. Myomectomy procedure increase the chances of pregnancy and decreases the rate of spontaneous miscarriage in many studies ${ }^{6,7}$.

In practice, there is a paucity of data on this subject in our general population. Therefore, we have decided to determine the frequency of reproductive outcomes after abdominal myomectomy in infertile women in our general population. Our study will pave the way for our doctor community to improve the obstetric outcomes in infertile women with uterine fibroids.

\section{METHODOLOGY}

This case series included sixty women who underwent myomectomy to retain their capabilities of reproduction at Combined Military Hospital, Multan, from September 2015 to February 2017. Inclusion criteria were infertile women with uterine fibroids. Exclusion criteria were patients with history of endometriosis, ovarian cyst, and husband has abnormal semen analysis and adenomyosis. Routine investigation was done to all patients like ultrasound and hysterosalpingogram before and after the procedure. Informed consent was taken from all the participants after explaining risk and benefits. The procedure of abdominal myomectomy was done under general anesthesia. 
All fibroids were enucleated and large blood vessels were tied. Vicryl no 1 was used to close the uterine defect. After the procedure antibiotics as per protocol were given to all patients. All patients were followed up for 3, 6 and 12 months interval and data was collected for conception, spontaneous conception, and conception with assisted reproductive technique, live birth and miscarriage. Data was analyzed with statistical analysis program SPSS version 22 (SPSS, IBM). Qualitative variables like conception, type of conception and pregnancy outcomes were computed in term of frequency and percentage. Mean with standard deviation was presented for quantitative variables like age, number of fibroids and size of fibroids.

\section{RESULTS}

These interventional studies included 60 women who underwent myomectomy to retain their capabilities of reproduction at Combined Military Hospital, Multan. Mean age was $27.33 \pm 4.03$ years, Mean number of fibroid was $1.36 \pm 0.48$ and mean size of fibroid was $7.06 \pm 1.64 \mathrm{~cm}$ (table-I). Out of 60 patients who went under myomectomy, 28 (46.7\%) women had conceived after myomectomy (figure). Out of 28 patients who conceived $26(92.9 \%)$ had spontaneous conception while $2(7.1 \%)$ had conception by assisted reproductive technique (table-II). Live birth was seen in 22 (78.6\%)

Table-I: Mean age, number of fibroids and size of fibroids $(n=60)$.

\begin{tabular}{|c|c|c|}
\hline \multicolumn{2}{|l|}{ Demographics } & Mean \pm SD \\
\hline \multicolumn{2}{|l|}{ Age (years) } & $27.333 \pm 4.03$ \\
\hline \multicolumn{2}{|l|}{ Number of Fibroids } & $1.366 \pm 0.48$ \\
\hline \multicolumn{2}{|c|}{ Size of Fibroids (cm) } & $7.066 \pm 1.64$ \\
\hline \multicolumn{3}{|c|}{$\begin{array}{l}\text { Table-II: Distribution of patients according to type of } \\
\text { conception }(n=28) \text {. }\end{array}$} \\
\hline Type of Conception & No. of Patients & Percentage \\
\hline Spontaneous & 26 & 46.7 \\
\hline $\begin{array}{l}\text { Assisted reproductive } \\
\text { technique }\end{array}$ & 2 & 7.2 \\
\hline
\end{tabular}

conceived women while $6(21.4 \%)$ women had miscarriage (figure).

\section{DISCUSSION}

Uterine fibroids are common in women of reproductive age. The influence of myomas on reproduction has been clearlry demonsterated and the appearance of the uterine fibroids been linked to infertility. Definitive surgery for uterine leiomyomas has long been criticized, but conservative interventions are not universally accepted either, even for the infertile women $^{1,2}$. Morbidity, complications, the possibility of iatrogenic damage to the reproductive organs, and the

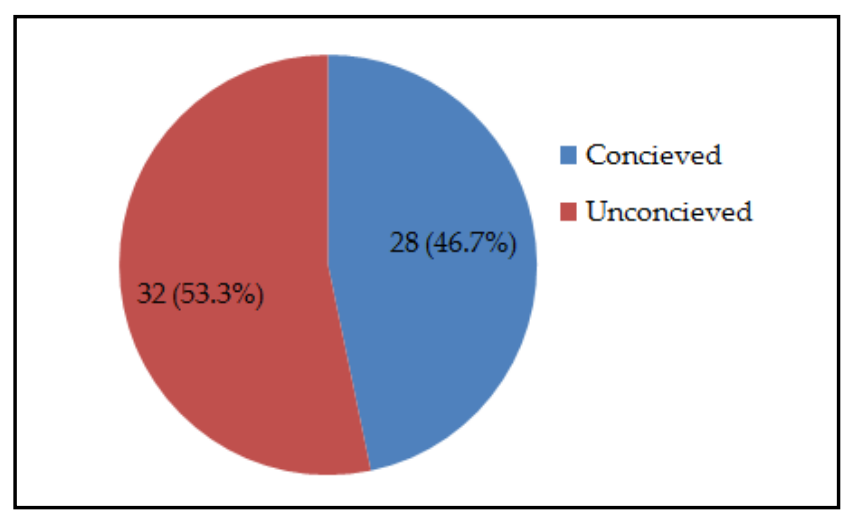

Figure: Distribution and percentage of patients according to conception $(n=60)$.

increased risk of a cesarean section in case of delivery after the operation all play role in limiting the consensus on myomectomy ${ }^{3,4}$, but the results of our study show that abdominal myomectomy has a favorable impact on fertility outcomes by enhancing fertility. There was $78.6 \%$ successful live birth after procedure which is a clear indication that myomectomy should be a standard procedure and treatment in women with uterine fibroids.

Somewhat surprisingly and at odds with the recent findings of Eldar-Geva et al anatomic characteristics of the fibroid (number, size and location with respect to both the uterine wall and body) did not significantly influence the post-operative probability of conception $^{5}$. This seems to militate against a potential casual relation between fibroids and infertility because a gradient effect of the major anatomic variable of the lesion apparently was lacking. More over the determinants that had a substantial impact on the out come of the surgery were independent of the treatment (i.e. age, infertility and presence of additional but treatable in fertility factors on the other hand the cumulative pregnancy rate of the population studied is well above that observe in couples with un-explained infertility who are followed up without treatment ${ }^{6,7}$. The study findings were consistent with other studies 8,9 . In one study successful live birth was shown $75 \%$ after abdominal myomectomy ${ }^{10}$. A recent review of 27 studies by Vercellini et al, found no conclusive evidence whether the number, size, or location of fibroids prior to myomectomy influenced postoperative pregnancy rates ${ }^{11,12}$. In a Pakistani studies the percentage of successful live birth after this procedure was $50 \%$, in our study conception rate was $46.7 \%$, in which $92.9 \%$ patients conceived spontaneously. Age was found to have a clinically significant impact on postoperative pregnancy 
rate, with a lower spontaneous or assisted conception pregnancy rate over 35 years ${ }^{13,14}$.

It has been suggested that surgical management of fibroids improve fertility rates and reduce miscarriage rates, but the results of surgical intervention for each type of fibroids may vary. It is well accepted that sub-mucosal, intramural and sub-serosal fibroids are being in decreasing order of importance in causing infertility ${ }^{16-18}$. Fibroids more than $5 \mathrm{~cm}$ in diameter and those located near the cervix or the tubal ostia are more likely to cause problem. Moreover the fibroids which are distorting the endrometrial cavity may create the abnormal site for placental implantation and growth resulting impaired fertility and an increased risk of spontaneous miscarriage, preterm laour and delivery ${ }^{19}$, ion and In this study the miscarriage rate was $21.4 \%$, which were comparable with a study. Abdominal approach for myomectomy was adopted in this study, which raised the concern of uterine rupture in study population during pregnancy, so 24 (92.3\%) women delivered by elected cesarean section and $2(7.7 \%)$ by vaginal delivery in case of subserosa 1 myomectomy. Uterine rupture is a major concern for all those women who conceived after myomectomy, incidence of uterine rupture was not seen in our study. Suture technique during myomectomy decrease the risk of uterine rupture by using interrupted sutures with vicryl no. 1 in the myoma bed, which provides homeostasis and decrease the risk of uterine rupture during pregnancy in the long term ${ }^{20}$. The safety of other treatment possibilities such as myolysis using electrical, thermal ultrasound energy for women desiring pregnancy is unknown and need further studies to determine.

\section{CONCLUSION}

Abdominal myomectomy should be the standard treatment of infertile women with uterine fibroids if no other underlines cause of infertility. Moreover, this study results also showed that younger patients might be benefited more in term of reproductive outcomes after abdominal myomectomy.

\section{CONFLICT OF INTEREST}

This study has no conflict of interest to be declared by any author.

\section{REFERENCES}

1. Day Baird D, Dunson DB, Hill MC, Cousins D, Schectman JM. high cumulative incidence of uterine leiomyoma in black and white women: ultrasound evidence. Am J Obstet Gynecol 2003; 188(1): 100-7.

2. Vercellini P. Uterine adenomyosis and in vitro fertilization outcome: A systematic review and meta- analysis. Human Reproduction, 2014; 29(5): 964-7.

3. You JH, Sahota DS, Yuen PM. Uterine artery embolization, hysterectomy, or myomectomy for symptomatic uterine fibroids: a costutility analysis. Fertil Steril 2009; 91(2): 580-8.

4. Eldar-Geva T, Meagher S, Healy DL, MacLachlan V, Breheny S, Wood C. Effect of intramural, subserosal, and submucosal uterine fibroids on the outcome of assisted reproductive technology treatment. Fertil Steril 1998; 70(6): 87-91.

5. Sinclair D, Gaither K, Mason TC. Fertility outcomes following myomectomy in an urban hospital setting. J Nat Med Assoc 2005; 97(10): 1346-8.

6. Rovio PH, Heinonen PK. Pregnancy outcomes after transvaginal myomectomy by colpotomy. Eur J Obstet Gynaecol Reprod Biol 2012; 161(2): 130-3.

7. Tsuji I, Fujinami N, Kotani Y, Tobiume T, Aoki M, Murakami K. Reproductive outcome of infertile patients with fibroids based on the patient and fibroid characteristics; optimal and personalized management. Gynecol Obstet Invest 2016; 81(4): 325-32.

8. Annette I, La Morte, Lalwani S, Diamond M. Morbidity associated with abdominal myomectomy. Am J Obstet Gynecol 1993; 82(3): 897-00.

9. Kunde DK, Khalaf Y. Morbidity of abdominal myomectomy: dispelling the myth. Gynecol Surg 2005; 5(2): 82-88.

10. Iftikhar R. Outcome of abdominal myomectomy. J Surg Pak 2009; 14(4): 85-8.

11. Verkauf BS. Myomectomy for fertility enhancement and preservation. Fertil Steril 1992; 58(1): 1-15.

12. Machupalli S, Norkus EP, Mukherjee TK, Reilly KD. Abdominal myomectomy increases fertility outcome. Gynecol Obstet 2013; 3(144): 2161-2.

13. Verkauf VS. Myomectomy as a fertility promoting procedure. Infertility and reproductve medicines clinics of North America 1996; 156(2): 319-22.

14. Pitter MC, Gargiou AR, Bonaventura LM, Stefano Lehman J, Srouji SS. Pregnancy outcomes following robot-assisted myomectomy. Human Reprod 2013; 28(1): 99-8.

15. Lonnerfors C, Persson J. Pregnancy following robot-assisted laparoscopic myomectomy in women with deep intramurals myomas. Acta Obstetrics et Gyn aecological Scandinavica, 2011; $99(9)$ : 972-77.

16. Spies JB, Bradley LD, Guido R, Maxwell GL, Levine BA, Coyne K. Outcomes and Gynaecolo J 2010; 116(3): 641-52.

17. Malzoni M, Rotondi M. Fertility after laparoscopic myomectomy of large uterine myomas: operative techniques and preliminary results. European J Gynaecol Oncol 2003; 24(1): 79-82.

18. Seracchioli R, Roosi F, Govoni S. Fertility and obstetric outcome after laparoscopic myomectomy of large myomata: a randomized comaparison with abdominal myomectomy. Human Reprod 2000; 15(12): 2663-68.

19. Hurst BS, Matthews ML, Marshburn PB. Laproscopic myomectomy for symptomatic uterine myomas. Fertil Steril 2005; 83(1): $1-23$.

20. Ramzy AM, Sattar M, Amin Y, Mansour RT, Serour GI, Aboulghar MA. Uterine mymota and outcome of assisted reproduction. Hum Retrod 1998; 3(2): 198-2. 